\title{
Optical amplification and stability of spiroquaterphenyl compounds and blends
}

T. Fuhrmann-Lieker

th.fuhrmann@uni-kassel.de

\section{J. Lambrecht}

\section{N. Hoinka}

M. Kiurski

\section{A. Wiske \\ G. Hagelstein}

N. Yurttagül

\section{Abdel-Awwad}

H. Wilke

F. Messow

\section{H. Hillmer}

\section{J. Salbeck}

University of Kassel, Macromolecular Chemistry and Molecular Materials, Institute of Chemistry and Center for Interdisciplinary Nanoscience and Technology, D-34109 Kassel, Germany

University of Kassel, Macromolecular Chemistry and Molecular Materials, Institute of Chemistry and Center for Interdisciplinary Nanoscience and Technology, D-34109 Kassel, Germany

University of Kassel, Macromolecular Chemistry and Molecular Materials, Institute of Chemistry and Center for Interdisciplinary Nanoscience and Technology, D-34109 Kassel, Germany

University of Kassel, Macromolecular Chemistry and Molecular Materials, Institute of Chemistry and Center for Interdisciplinary Nanoscience and Technology, D-34109 Kassel, Germany

University of Kassel, Macromolecular Chemistry and Molecular Materials, Institute of Chemistry and Center for Interdisciplinary Nanoscience and Technology, D-34109 Kassel, Germany

University of Kassel, Macromolecular Chemistry and Molecular Materials, Institute of Chemistry and Center for Interdisciplinary Nanoscience and Technology, D-34109 Kassel, Germany

University of Kassel, Macromolecular Chemistry and Molecular Materials, Institute of Chemistry and Center for Interdisciplinary Nanoscience and Technology, D-34109 Kassel, Germany

University of Kassel, Technological Electronics, Institute of Nanostructure Technologies and Analytics and Center for Interdisciplinary Nanoscience and Technology, D-34109 Kassel, Germany

University of Kassel, Technological Electronics, Institute of Nanostructure Technologies and Analytics and Center for Interdisciplinary Nanoscience and Technology, D-34109 Kassel, Germany

University of Kassel, Technological Electronics, Institute of Nanostructure Technologies and Analytics and Center for Interdisciplinary Nanoscience and Technology, D-34109 Kassel, Germany

University of Kassel, Technological Electronics, Institute of Nanostructure Technologies and Analytics and Center for Interdisciplinary Nanoscience and Technology, D-34109 Kassel, Germany

University of Kassel, Macromolecular Chemistry and Molecular Materials, Institute of Chemistry and Center for Interdisciplinary Nanoscience and Technology, D-34109 Kassel, Germany

In this contribution, we present a systematic investigation on a series of spiroquaterphenyl compounds optimised for solid state lasing in the near ultraviolet (UV). Amplified spontaneous emission (ASE) thresholds in the order of $1 \mu \mathrm{J} / \mathrm{cm}^{2}$ are obtained in neat (undiluted) films and blends, with emission peaks at $390 \pm 1 \mathrm{~nm}$ for unsubstituted and meta-substituted quaterphenyls and $400 \pm 4 \mathrm{~nm}$ for para-ether substituted quaterphenyls. Mixing with a transparent matrix retains a low threshold, shifts the emission to lower wavelengths and allows a better access to modes having their intensity maximum deeper in the film. Chemical design and blending allow an independent tuning of optical and processing properties such as the glass transition.

[DOI: http://dx.doi.org/10.2971/jeos.2015.15007]

Keywords: Amplified spontaneous emission, lasers, organic materials, optical materials, waveguides, threshold

\section{INTRODUCTION}

Spirooligophenyls that are characterised by a spiro linkage of two oligophenyl chromophores have been proposed as efficient organic solid state laser materials [1]-[3]. Due to the large Stokes shift with reduced self-absorption and the low tendency towards crystallisation, they exhibit low thresholds for stimulated emission in thin films. First-order Distributed Feedback (DFB) lasers have been realized with these materials [4]-[6]. A recent major breakthrough in the field of organic lasers, albeit in other material systems, was the demonstration of pumping schemes using inexpensive inorganic diode lasers [7]-[9] which arises more interest for tunable UV emit- ter materials. Currently, alternative laser geometries for organic solid state lasers are exploited, for instance vertically emitting stacks with Bragg mirrors [10]. In combination with diode laser pumping, small spectroscopic detection systems for analytical applications may be envisaged for arrays of vertical organic lasers. If the material is distributed in a number of layers for higher gain, however, the absorption of the active material should be not too high ensuring a sufficient penetration depth into the cavity.

For the various types of horizontally and vertically emitting 


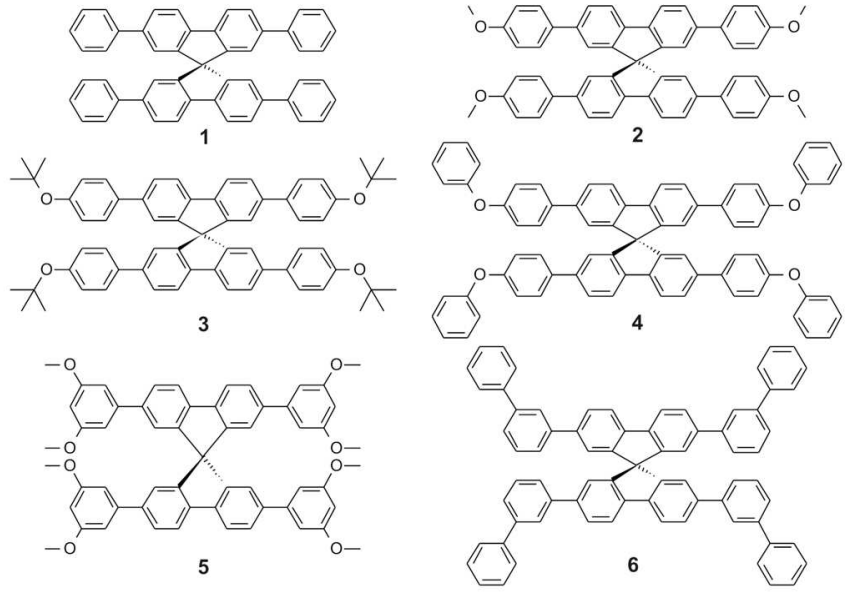

FIG. 1 Chemical structures of spiroquaterphenyl compounds used as emitters.

lasers, specially adapted profiles for the laser-active materials have to be fulfilled. In addition to optical properties, processing issues have to be regarded as well. For instance, in special vertical emitting structures for which the organic material has to be infiltrated [11] into void gaps from the melt or supercooled liquid, the glass transitions and melting point should be not too high.

Chemical variation of the emitting compounds may easily adapt them for the purpose required. In this contribution we compare a series of novel spiroquaterphenyls for the suitability in distinct organic lasers. The compounds 2-5 (Figure 1) differ from the first generation of spiro compounds [1]-[6] by $\pi$-electron donating ether groups at different positions that introduces flexibility which is needed for better processing from the melt and may reduce brittleness in films on flexible substrates. Depending on the position (para or meta), the $\pi$-electron system is enlarged, shifting the optical transitions bathochromically, or not. One compound (6), exhibits the same conjugation length as the first-generation spiroquaterphenyl 1 but has the same molecular weight and spacefilling properties as the isomeric spiro-sexiphenyl [1, 2]. Key features for comparison of the compounds are optical threshold, measured for amplified spontaneous emission (ASE) in thin films, refractive index and absorption, as well as glass transition and melting points. Quaterphenyl is chosen as basic chromophore since it allows pumping in the UV below $360 \mathrm{~nm}$ while still emitting in the violet spectral range around $400 \mathrm{~nm}$. Further downconversion into the visible may then be achieved by Förster transfer $[12,13]$ if required.

\section{EXPERIMENTAL}

The synthesis of the materials based on Suzuki coupling reactions [14] is described in the appendix. Thin films were prepared by spincoating from a solution containing $15 \mathrm{mg}$ of the compound in $1 \mathrm{ml}$ chloroform (spectroscopic grade) onto glass substrates (VWR ISO 8037/1) at 3000 rpm. Thicknesses and optical data of the resulting films were determined by spectroscopic ellipsometry (Woollam VASE). ASE experiments were performed in a nitrogen-filled chamber with fused silica windows using a nitrogen laser as pump source (Lasertechnik Berlin MSG 800, 500 ps pulses at $10 \mathrm{~Hz}$ repetition rate). Spectra were recorded using a fiber spectrometer (Ocean Optics S2000) attached to the chamber. For thermal analyses, thermogravimetry (Perkin Elmer Diamond TG/DTA, rate $5 \mathrm{~K} / \mathrm{min}$ ) and differential scanning calorimetry (Perkin Elmer DSC7, rate $10 \mathrm{~K} / \mathrm{min}$ ) were applied. Static contact angles were measured with a KSV CAM100 system.

\section{RESULTS AND DISCUSSION}

The structure of the investigated solid state quaterphenyl compounds can be found in Figure 1. In order to facilitate processing from the liquid state, conformational flexibility was introduced by either substitution in para position (2-4) with different ether groups, varying in bulkiness and influencing the conjugated system due to the $\pi$-donor character, or by metasubstitution (5-6) with only a small influence on the $\pi$-system but changing the morphological properties.

\subsection{Stimulated emission and optical properties of neat films}

In order to achieve best performances but allow a comparable screening of the materials, films were prepared in a thickness range around $100 \mathrm{~nm}$ that allows only one TE mode propagating in the film at the emission wavelength. Reaching the threshold intensity by optical pumping at $337 \mathrm{~nm}$, the emission spectrum narrows until only a narrow ASE line with a width of a few nanometers remains, indicating amplified spontaneous emission. The threshold incident intensity was evaluated according the procedure described in ref. [2]. Table 1 comprises the samples with the lowest threshold obtained for each material, evaluated from a number of samples (4-6) with a thickness variation of $10-20 \%$. Of course, the amount of absorbed energy varies with the absorption spectrum of the respective material and also the reflection coefficient at the surface, therefore the real and imaginary part of the refractive index at the pumping wavelength of $337 \mathrm{~nm}$ (and also for $355 \mathrm{~nm}$ ) are included in the table. All dispersion curves were modelled by a combination of isotropic Gaussian and Tauc-Lorenz [15] oscillators, fitting the ellipsometric data well.

All compounds exhibit ASE with thresholds in the order of $1 \mu \mathrm{J} / \mathrm{cm}^{2}$, with the exception of PhO-Sp $4 \Phi$, showing a slightly higher threshold. The experimental error due to the fitting procedure is estimated to be a factor of 2, therefore smaller differences are not to be considered as significant. The threshold are well within the range that has been obtained by the same method for other spiro compounds with longer emission wavelengths [2, 3], showing that low ASE thresholds are a general feature of spirooligophenyls irrespective of the substitution. Since the geometry of ASE waveguides and DFB lasers are different, threshold values cannot be directly compared with DFB laser thresholds. Usually, however, the DFB laser thresholds as in ref. [4]-[7] are one order of magnitude higher with the same material, despite of the better mode confinement in these devices. Gain and threshold measurements on a subset of the investigated compounds under different pumping conditions ( $355 \mathrm{~nm}, 10 \mathrm{~ns}$ pulses, higher pulse energies up to $3000 \mu \mathrm{J} / \mathrm{cm}^{2}$ ) are reported elsewhere [16]. In comparison 


\begin{tabular}{|c|c|c|c|c|c|c|c|}
\hline Compound & $\lambda_{\mathrm{ASE}} / \mathrm{nm}$ & $I_{t h} / \mu \mathrm{J} \mathrm{cm}^{-2}$ & $\mathrm{FWHM} / \mathrm{nm}$ & $d / \mathrm{nm}$ & $\begin{array}{l}n_{\mathrm{ASE}} \\
k_{\mathrm{ASE}}\end{array}$ & $\begin{array}{l}n_{337} \\
k_{337}\end{array}$ & $\begin{array}{l}n_{355} \\
k_{355}\end{array}$ \\
\hline $1 \mathrm{Sp} 4 \Phi$ & 39 & 2.2 & 5.0 & 111 & $\begin{array}{l}1.991 \\
0.001\end{array}$ & $\begin{array}{l}1.96 \\
0.64\end{array}$ & $\begin{array}{l}2.40 \\
0.26\end{array}$ \\
\hline $2 \mathrm{MeO}-\mathrm{Sp} 4 \Phi$ & 404 & 2.1 & 3.1 & 77 & $\begin{array}{l}1.977 \\
0.004\end{array}$ & $\begin{array}{l}1.89 \\
0.72\end{array}$ & $\begin{array}{l}2.16 \\
0.65\end{array}$ \\
\hline 3 tBuO-Sp4 $\Phi$ & 398 & 0.7 & 3.2 & 81 & $\begin{array}{l}1.825 \\
0.000\end{array}$ & $\begin{array}{l}1.75 \\
0.57\end{array}$ & $\begin{array}{l}2.14 \\
0.32\end{array}$ \\
\hline 4 PhO-Sp4 $\Phi$ & 401 & 8.7 & 3.6 & 92 & $\begin{array}{l}1.958 \\
0.004\end{array}$ & $\begin{array}{l}2.10 \\
0.36\end{array}$ & $\begin{array}{l}2.23 \\
0.37\end{array}$ \\
\hline $5(\mathrm{MeO})_{2}-\mathrm{Sp} 4 \Phi$ & 391 & 0.7 & 3.1 & 70 & $\begin{array}{l}1.862 \\
0.004\end{array}$ & $\begin{array}{l}1.91 \\
0.52\end{array}$ & $\begin{array}{l}2.11 \\
0.22\end{array}$ \\
\hline $6 m, p$-Sp6Ф & 390 & 1.2 & 3.9 & 71 & $\begin{array}{c}1.858 \\
0.13\end{array}$ & $\begin{array}{l}2.00 \\
0.36\end{array}$ & $\begin{array}{l}2.03 \\
0.09\end{array}$ \\
\hline
\end{tabular}

TABLE 1 From left to right: Compound, peak wavelength, minimum ASE threshold incident intensity, full width at half maximum of ASE spectrum, film thickness $d$ corresponding to threshold value, real $(n)$ and imaginary $(k)$ part of the refractive index for ASE wavelength, $337 \mathrm{~nm}$ and $355 \mathrm{~nm}$, respectively.

with irradiation at $355 \mathrm{~nm}$, the thresholds measured here at $337 \mathrm{~nm}$ are at least one order of magnitude lower. This can be attributed to the shorter pulse length in agreement with ref. [17], a better absorption at $337 \mathrm{~nm}$ (that is however not a sufficient condition as the discussion in Section 3.3 about the relation between penetration depth and gain demonstrates) as well as to the different substrate (silicon with thermal oxide was used in ref. [16], glass in the measurements presented here). For the samples discussed here, the lowest threshold values were obtained for $t \mathrm{BuO}-\mathrm{Sp} 4 \Phi$ and $(\mathrm{MeO})_{2}-\mathrm{Sp} 4 \Phi$. The ASE peak wavelengths reflect the substitution pattern: ether substitution in para position shifts the ASE peak with respect to $\operatorname{Sp} 4 \Phi$ bathochromically by about $10 \mathrm{~nm}$, whereas meta substitution retains the ASE peak in the UV at around $390 \mathrm{~nm}$.

With respect to emission stability, the compounds show different behaviour. For stability measurements, the films are exposed in a nitrogen atmosphere to 500 ps pulses at $10 \mathrm{~Hz}$ repetition rate with pulse energy densities of $613 \mu \mathrm{J} / \mathrm{cm}^{2}$. In Figure 2 , the integrated area under the emission spectrum is plotted against irradiation time for the compounds. In all cases, at the beginning all plots show a significant decrease of the signal until a plateau is reached the decrease of which is on a larger timescale or not detectable. Fitting a monoexponential decay leads to systematic deviations, therefore a more complex degradation mechanism is assumed. Taking the times at which the signal decreased by half the value for comparison, the stability increases in the order $(\mathrm{MeO})_{2}-\mathrm{Sp} 4 \Phi(18 \mathrm{~s})<$ tBuO-Sp4 $\Phi$ (360 s) < MeO-Sp4Ф (600 s) < PhO-Sp4 $\Phi$ (800 s) $<m, p$-Sp6Ф (2000 s) < Sp4 $\Phi$ (6000 s). Morphological alterations [16] and photochemical reactions [18] are suggested as possible mechanisms responsible for this degradation. Since the emission spectra in the plateau exhibits normal fluorescence we conclude that the degradation of ASE at the beginning is caused by morphological alterations in the films that reduce the gain. For $(\mathrm{MeO})_{2}-\mathrm{Sp} 4 \Phi$, but not for the other compounds, crystallization of the samples was observed. Generally, the ether substituted compounds are less stable than the pure hydrocarbons, but a correlation to thermal properties (see below) cannot be found. The remaining photoluminescence signal is fairly constant, thus photobleaching may occur only on a larger timescale.

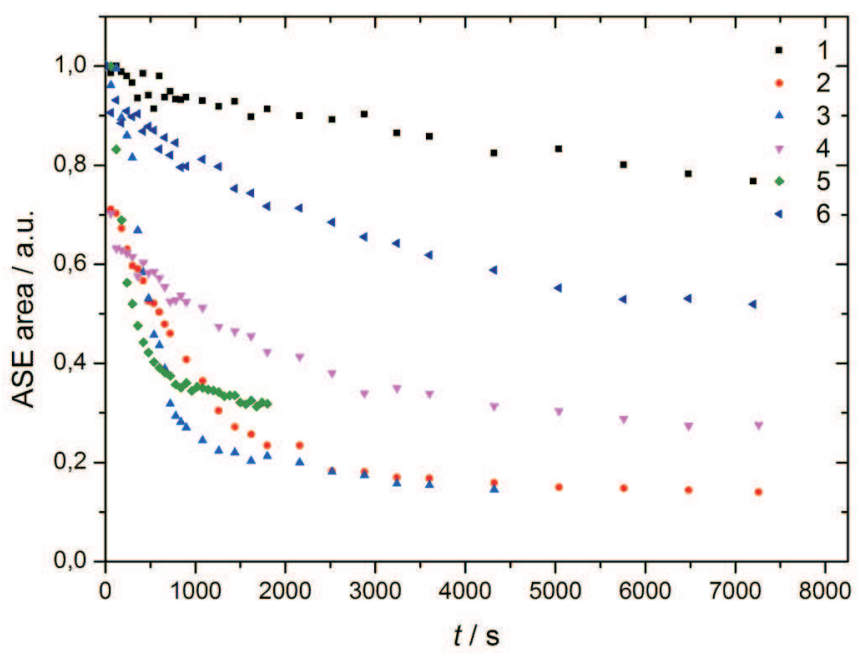

FIG. 2 Relative stability of the ASE (integral area under spectrum) measured after a number of shots. 1 second corresponds to 10 pulses at $613 \mu \mathrm{J} / \mathrm{cm}^{2}$.

\subsection{Processing properties}

The optical compounds differ in their glass transition and melting behaviour (Table 2). The following points are noticeable: In contrast to the other compounds, $(\mathrm{MeO})_{2}-\mathrm{Sp} 4 \Phi$ crystallises upon cooling at $10 \mathrm{~K} / \mathrm{min}$, and no glass transition was detected. The two-fold ether substitution antagonises the core rigidity and makes the molecule too flexible for forming a stable glass. The tendency towards crystallization can also be seen as the origin for the short ASE lifetime as discussed above. The other meta-substituted compound, $m, p-S p 6 \Phi$, has a low ratio between $T_{g}$ and $T_{m}$ demonstrating a high conformational flexibility, but the optical stability is substantially better. Lowest values for both $T_{g}$ and $T_{m}$ in combination with good optical stability and moderate threshold are obtained for $\mathrm{PhO}-\mathrm{Sp} 4 \Phi$ which makes it a good candidate for infiltration processing. The best morphological stability in terms of a high $T_{g}$ and a high ratio between $T_{g}$ and $T_{m}$ was obtained for $t \mathrm{BuO}-\mathrm{Sp} 4 \Phi$, but in this case, infiltration from the melt cannot be done, since it decomposes above the melting point, with the lowest decomposition temperature of all compounds. As it is deduced from a step-wise loss of weight of $23.5 \%$ at decomposition, it cleaves at the alkyl-oxygen bond leaving 
2,2',7,7'-tetra(4-hydroxyphenyl)-9,9'-spirobifluorene which is confirmed by mass spectrum $(m / z=684.39)$ and NMR analysis of the residue.

Wetting behaviour is important for the stability of the films. For three compounds, it was possible to measure the contact angle of a molten droplet on silicon substrates with $200 \mathrm{~nm}$ thermal oxide which is comparable to the glass used in the ASE experiments. Good adhesion to this hydrophilic substrate exhibits MeO-Sp4 $\Phi$ with a contact angle of $16^{\circ}$, followed by Sp4 $\Phi\left(24^{\circ}\right)$, and finally PhO-Sp4 $\Phi\left(57^{\circ}\right)$. Hydrophobic substrate pretreatment with hexa(methylen)disilazane (HMDS) does not provide better adhesion: the values change to $42^{\circ}$, $47^{\circ}$, and $61^{\circ}$, respectively. Dewetting may be an issue for the more hydrophobic compounds at long storage times, however, no correlation to the ASE degradation measurements can be found, thus it seems not to contribute to the degradation process.

\subsection{Independent optimization of optical performance and processing by blending}

One of the advantages of organic optical materials is the possibility of tuning the properties not only by chemical functionalization but also by blending [17], [19]-[21]. In contrast to polymers, low-molecular glasses can be easily mixed since the entropy of mixing is quite large. In order to investigate the effect of dilution in mixtures of spiro compounds, $\mathrm{Sp} 4 \Phi$ and $t$ BuO-Sp $4 \Phi$ were separatedly blended into 3,3',6,6'-Tetrakis(biphenyl-3-yl)-9,9'-spirobifluorene ( $m, m$-Sp6 $\Phi)$ as matrix by spincoating from a mixed solution. The matrix compound, cited in ref. [22] with $T_{g}=413 \mathrm{~K}$ and known optical data, has only biphenyl chromophores in direct conjugation and thus does not absorb in the same spectral region as the quaterphenyls. A ratio of $5 \%$ spiroquaterphenyl compound was chosen since this ratio provided lowest threshold values in experiments mixing spiro-sexiphenyl with this matrix [22]. In both cases considered here, the ASE wavelength in the blend was lower as in the neat film (383 nm for 5\% Sp4 $\Phi$ in $m, m-\mathrm{Sp} 6 \Phi, 394 \mathrm{~nm}$ for $t \mathrm{BuO}-\mathrm{Sp} 4 \Phi$ in $m, m-\mathrm{Sp} 6 \Phi)$, whereas the threshold was in the same order of magnitude within experimental errors $\left(2.4 \mu \mathrm{J} / \mathrm{cm}^{2}\right.$ for a $84 \mathrm{~nm}$ thick film of Sp4 $\Phi$ in $m, m-S p 6 \Phi$ and $0.4 \mu \mathrm{J} / \mathrm{cm}^{2}$ for a $81 \mathrm{~nm}$ thick film of $t \mathrm{BuO}-\mathrm{Sp} 4 \Phi$ in $m, m-\mathrm{Sp} 6 \Phi$, respectively). The hypsochromic shift of the emission can be attributed to lower losses at the short-wavelength side of the emission band due to lower selfabsorption since the matrix contributes less at the pumping

\begin{tabular}{|l|c|c|c|c|}
\hline Compound & $T_{g} / \mathrm{K}$ & $T_{m} / \mathrm{K}$ & $T_{g} / T_{m}$ & $T_{d} / \mathrm{K}$ \\
\hline 1 Sp4 $\Phi$ & 441 & 565 & 0.78 & 733 \\
\hline 2 MeO-Sp4 $\Phi$ & 425 & 527 & 0.81 & 724 \\
\hline 3 $t$ BuO-Sp4 $\Phi$ & 465 & 552 & 0.84 & 601 \\
\hline 4 PhO-Sp4 $\Phi$ & 389 & 516 & 0.75 & 798 \\
\hline 5 $(\mathrm{MeO})_{2}-S p 4 \Phi$ & - & 602 & - & 716 \\
\hline 6 $m, p-S p 6 \Phi$ & 404 & 574 & 0.70 & 707 \\
\hline
\end{tabular}

TABLE 2 Glass transition temperature $T_{g}$, melting point $T_{m}$ (onset) and decomposition temperature $T_{d}$ (for $5 \%$ loss of weight) of the compounds. wavelength $\left(n_{337}=2.07, k_{337}=0.05\right)$. That the threshold is not increased even if the chromophore is diluted by a factor of 20 may be surprising at first sight, but can be readily understood if the distribution of excitation density within the film is considered (Figure 3). Due to the limited penetration depth of the pumping light into the film, mainly the molecules near to the surface are excited. This is an important difference to semiconductor lasers in which a homogenous excitation density is often assumed. For efficient coupling to the TE waveguide mode that has its intensity maximum deeper in the film, a higher penetration depth is favourable for stimulated emission. The correct modal gain is described as [23]

$$
g_{m o d}^{T E}=\frac{\sum_{i} n_{i} g_{i} \int_{i} E_{y}^{2} d x}{n_{e f f} \int_{-\infty}^{+\infty} E_{y}^{2} d x}=\sum_{i} \frac{n_{i}}{n_{e f f}} \Gamma_{i} g_{i}
$$

summing over all layers $i$ with their respective confinement factors $\Gamma_{i}$ of the transverse electrical field $E_{y}$ of the mode and their local gain $g_{i}$. Here, ni denotes the local refractive index, and $n_{\text {eff }}$ the mode index at the ASE wavelength. If the local gain is an exponentially decreasing function of the penetration depth $x$ due to absorption, the gain factor $\gamma$ as ratio between modal gain and material gain at the incident intensity (i.e. directly at the surface) has to expressed by the integral

$$
\gamma=\frac{g_{\text {mod }}^{T E}}{g\left(I_{0}\right)}=\frac{n_{\text {active }}}{n_{\text {eff }}} \frac{\int_{\text {active }} E_{y}^{2} \exp \left(-4 \pi k_{337} x / 337 \mathrm{~nm}\right) d x}{\int_{-\infty}^{+\infty} E_{y}^{2} d x}
$$

This integral corresponds to the area marked red in Figure 3. For a better illustration of the effect of penetration depth, the calculations were made with the same mode profile. However, the modes shift slightly in the matrix since the refractive index of the film can be assumed as similar to the matrix $\left(n_{383}=1.89, n_{394}=1.87\right)$. Experimental values are $n_{383}=1.898$ for Sp4 $\Phi$ in $m, m-S p 6 \Phi$ and $n_{394}=1.857$ for $t$ BuO-Sp4 $\Phi$ in $m, m-\operatorname{Sp} 6 \Phi$ which is in agreement with an effective medium approximation, the refractive index of $\operatorname{Sp} 4 \Phi$ being higher and that of $\mathrm{tBuO}-\mathrm{Sp} 4 \Phi$ being lower than that of the matrix. Also, the transmission factor at the film surface differs slightly because of the different refractive indices at the pumping wavelength, but values do not deviate much from approximately $88 \%$. For the generic model in Figure 3 with a specified thickness of $100 \mathrm{~nm}$, the gain factor $\gamma$ for the TE mode is $25 \%$ in the neat film, but as high as $63 \%$ in the diluted film. Table 3 summarizes the gain factors of neat and doped films with the same active materials used in our experiments. These values demonstrate the advantage of pumping in a wavelength and concentration range in which the absorption is not too high. By more efficient coupling to the ASE mode and less reabsorption, the needed amount of active material can be reduced substantially.

\section{CONCLUSIONS}

A series of quaterphenyl compounds have been shown as suitable for stimulated emission in the solid state with low thresholds. By different chemical substitutions, emission and processing properties can be tuned for particular laser applications. Blending with a transparent matrix is advantageous 

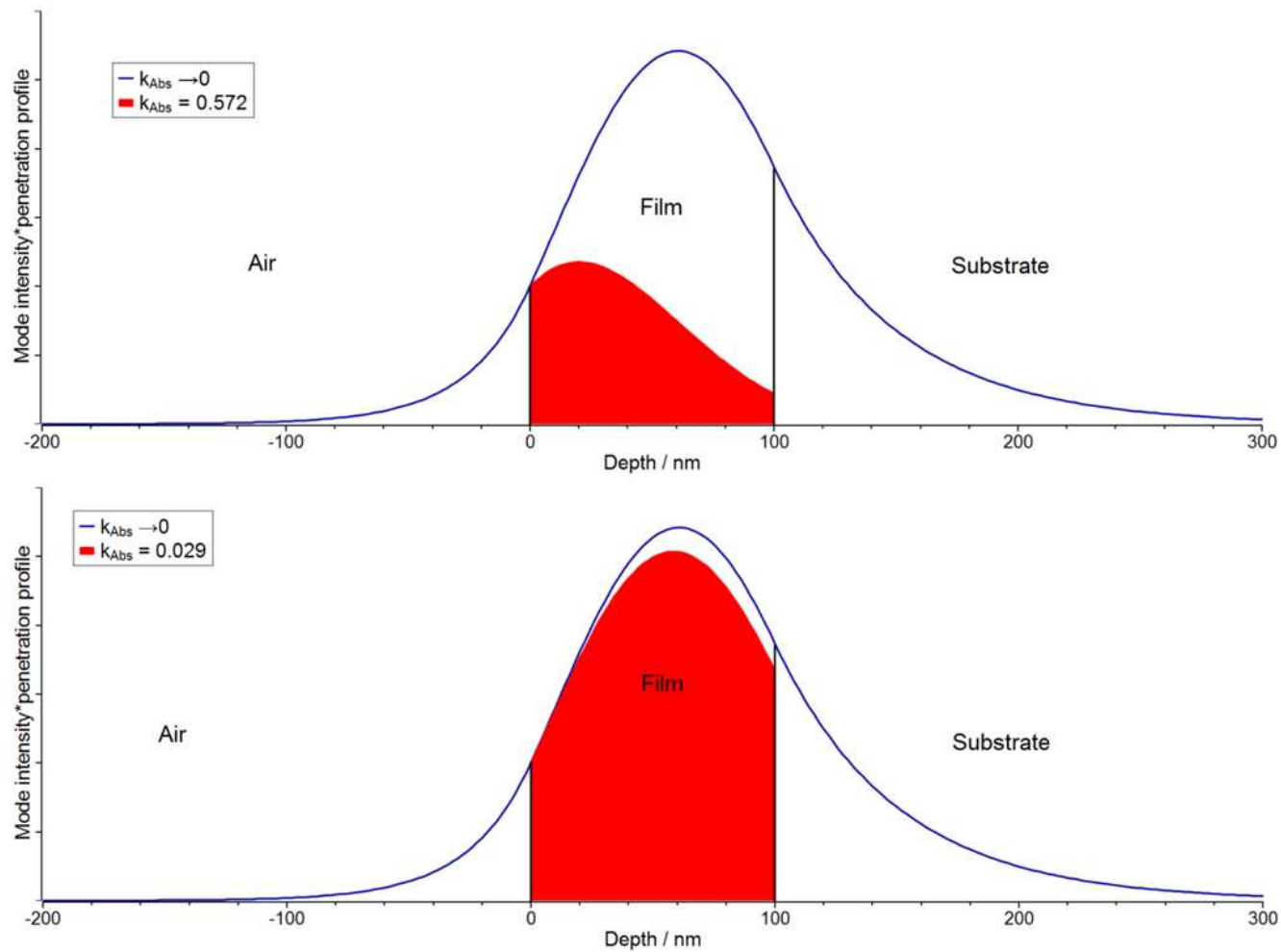

FIG. 3 Mode intensity profile (transverse electrical field, blue line) and gain factor (red area) for a neat film with high absorption (upper graph) and a film in which the chromophore is diluted by a factor of 20 (lower graph). Only if absorption is low ( $k_{\text {Abs }} \rightarrow 0$ ) the gain factor corresponds to the confinement factor given by the area of the blue curve in the active film, assuming a homogeneously pumped material. A mean refractive index of $n_{\text {active }}=1.87$ of the active material and a film thickness of 100 nm leading to a mode effective index of $n_{\text {eff }}=1.60$ are assumed in both cases for better comparison. The ratio $n_{\text {active }} / n_{\text {eff }}$ has to be taken into account in calculating the absolute values of the gain factor.

\begin{tabular}{|l|c|c|c|c|c|c|c|}
\hline Sample & $\lambda_{A S E} / \mathrm{nm}$ & $I_{t h} / \mu \mathrm{J} \mathrm{cm}-2$ & $d / \mathrm{nm}$ & $n_{\text {ASE }}$ & $n_{\text {eff }}$ & $k_{337}$ & $\gamma$ \\
\hline Sp4 $\Phi$ & 390 & 2.2 & 111 & 1.991 & 1.745 & 0.64 & $26.8 \%$ \\
\hline Sp4 $\Phi / m, m-S p 6 \Phi$ & 383 & 2.4 & 84 & 1.898 & 1.609 & 0.032 & $40.0 \%$ \\
\hline$t$ BuO-Sp4 $\Phi$ & 398 & 0.7 & 81 & 1.825 & 1.552 & 0.57 & $21.0 \%$ \\
\hline$t$ BuO-Sp4 $\Phi / m, m-S p 6 \Phi$ & 394 & 0.4 & 81 & 1.857 & 1.570 & 0.0285 & $53.5 \%$ \\
\hline
\end{tabular}

TABLE 3 Comparison of neat and doped films. From left to right: Sample, peak wavelength, minimum ASE threshold incident intensity, film thickness, measured refractive index of film, calculated effective mode index (TE), extinction coefficient at pumping wavelength, calculated gain factor.

since it allows a larger penetration depth and independent tuning of processing properties. For instance, the glass transition depends on the glass transition temperatures of both components, $T_{g, 1}$ and $T_{g, 2}$, approximately following Fox' rule $1 / T_{g}=w_{1} / T_{g, 1}+w_{2} / T_{g, 2}[24], w_{i}$ being the mass fractions of the two components. As our experiments show, the threshold is not deteriorated by dilution in thin film amplifiers, and in vertical emitting structures the advantages of a larger penetration depth can be expected to have even a higher impact on efficiency.

\section{ACKN OWLEDGEMENTS}

We thank C. Herb for the synthesis of $m, m-S p 6 \Phi$, T. Spehr for optical data, M. Maurer and E. Tatarov for NMR measurements and discussion, S. Fürmeier for mass spectroscopy, and B. Witzigmann for discussion within the project.

The project was funded by Deutsche Forschungsgemeinschaft, SA $438 / 11$, as part of a joint project together with HI $763 / 14$ and WI 3317/4.

\section{APPENDIX}

\section{A General procedure for the synthesis of $2,2^{\prime}, 7,7^{\prime}$-substituted spirobifluorenes used in this study}

A mixture of $1.0 \mathrm{mmol}$ 2,2,7,7-tetrabromo-9,9-spirobifluorene (CAS-No. 128055-74-3), $3.4 \mathrm{mmol}$ of the pinacol ester [14] of the appropriately substituted phenyl boronic acid, $7.6 \mathrm{mmol}$ potassium carbonate in $6.2 \mathrm{ml}$ toluene, $15 \mathrm{ml}$ tetrahydrofuran and $9 \mathrm{ml}$ water was placed in a Young flask with magnetic stirring bar. After degassing by nitrogen backfilling through a Pasteur pipette, the solution was stirred at room temperature for 15 minutes. Then $0.05 \mathrm{mmol} \mathrm{Pd}\left(\mathrm{PPh}_{3}\right)_{4}$ was added and the reaction was stirred at $80^{\circ} \mathrm{C}$. After 24 hours, another portion of $0.05 \mathrm{mmol} \mathrm{Pd}(\mathrm{PPh} 3) 4$ and $7.6 \mathrm{mmol}$ potassium carbonate was added, and the reaction mixture was stirred for further 48 hours. After cooling to room temperature, $43 \mathrm{mg} \mathrm{KCN}$ was added and the resulting solution was stirred until it became colourless. Organic and aqueous phases were separated and the aqueous one was washed with chloroform $(3 \times 20 \mathrm{ml})$. The 
combined organic phases were dried over $\mathrm{MgSO}_{4}$ and evaporated to dryness. The residue was purified by column chromatography over silica gel $(0.063-0.200 \mathrm{~mm}, \oslash 4 \mathrm{~cm} \times 35 \mathrm{~cm})$, eluent see below), yielding white solids.

\section{B Analytical data}

${ }^{1} \mathrm{H}-\mathrm{NMR}$ and ${ }^{13} \mathrm{C}-\mathrm{NMR}$ spectra were obtained with Varian MR-400, at $400 \mathrm{MHz}$ and $100 \mathrm{MHz}$ respectively. Chemical shifts $(\delta)$ are given in ppm. $\mathrm{CDCl}_{3}$ was used as solvent and internal reference. Mass spectra (MS-MALDI) were obtained with a Bruker Daltonics BiFlex IV, using DCTB as matrix.

a) 2,2',7,7'-Tetraphenyl-9, $\mathbf{9}^{\prime}$-spirobifluorene, Sp4 (1) was described before (CAS-No. 171408-92-7).

b) $2,2^{\prime}, 7,7^{\prime}$-Tetrakis-(4-methoxyphenyl)-9, $\mathbf{9}^{\prime}$-spirobifluorene, $\mathrm{MeO}-\mathrm{Sp} 4$ (2) is described in ref. [25].

c) 2,2',7,7'-Tetrakis-(4-tert-butoxyphenyl)-9,9'spirobifluorene, $t \mathrm{BuO}-\mathrm{Sp} 4$ (3):

Coupling reagent: pinacol ester of 4-tert-butoxyphenyl boronic acid, eluent: $\mathrm{CH}_{2} \mathrm{Cl}_{2}$ / hexane 1:1

Yield: $88 \%$ (purity $>99 \%$ )

${ }^{1} \mathrm{H}-\mathrm{NMR}: \delta=7.90(\mathrm{~d}, J=7.8 \mathrm{~Hz}, 4 \mathrm{H}), 7.61(\mathrm{~d}, J=8.0 \mathrm{~Hz}$, $4 \mathrm{H}), 7.34(\mathrm{~m}, 8 \mathrm{H}), 6.99(\mathrm{~s}, 4 \mathrm{H}), 6.91(\mathrm{~m}, 8 \mathrm{H}), 1.30(\mathrm{~s}, 36 \mathrm{H})$

${ }^{13} \mathrm{C}-\mathrm{NMR}: \delta=154.8,149.8,140.4,140.3,135.9,127.4,126.6$,

$124.2,122.4,120.2,78.6,66.2,28.8$

MS-MALDI: $m / z=908.94\left(\mathrm{M}^{+}\right)$

d) 2,2',7,7'-Tetrakis-(4-phenoxyphenyl)-9,9'-spirobifluorene, PhO-Sp4Ф (4):

Coupling reagent: 4-phenoxyphenyl boronic acid without previous esterification, eluent $\mathrm{CH}_{2} \mathrm{Cl}_{2}$ / hexane 2:1

Yield: $35 \%$ (purity $>99 \%$ )

${ }^{1} \mathrm{H}-\mathrm{NMR}: \delta=7.92(\mathrm{~d}, J=7.8 \mathrm{~Hz}, 4 \mathrm{H}), 7.62(\mathrm{dd}, \mathrm{J}=7.8 \mathrm{~Hz}$, $1.8 \mathrm{~Hz}, 4 \mathrm{H}), 7.40(\mathrm{~m}, 8 \mathrm{H}), 7.29(\mathrm{~m}, 8 \mathrm{H}), 7.06(\mathrm{~m}, 4 \mathrm{H}), 6.95$ $(\mathrm{m}, 16 \mathrm{H})$

${ }^{13}$ C-NMR: $\delta=157.1,156.6,149.8,140.4,140.3,136.0,129.7$,

$128.4,126.8,123.2,122.5,120.4,119.0,118.7,66.2$

MS-MALDI: $m / z=988.29\left(\mathrm{M}^{+}\right)$

e) 2,2',7,7'-Tetrakis-(3,5-dimethoxyphenyl)-9,9'-

spirobifluorene, $(\mathrm{MeO}) 2-\mathrm{Sp} 4 \Phi(5)$ :

Coupling reagent: pinacol ester of 3,5-dimethoxyphenyl boronic acid, eluent $\mathrm{CH}_{2} \mathrm{Cl}_{2}$

Yield: $43 \%$ (purity $>96 \%$ )

${ }^{1} \mathrm{H}-\mathrm{NMR}: 7.91(\mathrm{~d}, J=7.8 \mathrm{~Hz}, 4 \mathrm{H}), 7.61(\mathrm{dd}, J=7.7 \mathrm{~Hz}$, $1.5 \mathrm{~Hz}, 4 \mathrm{H}), 6.96(\mathrm{~d}, J=1.2 \mathrm{~Hz}, 4 \mathrm{H}), 6.57(\mathrm{~d}, J=1.9 \mathrm{~Hz}$, $8 \mathrm{H}), 6.35(\mathrm{t}, J=1.9 \mathrm{~Hz}, 4 \mathrm{H})$

${ }^{13}$ C-NMR: 160.9, 149.5, 143.2, 141.0, 141.9, 127.1, 122.9,

$120.3,105.6,98.9,66.2,55.4$

MS-MALDI: $m / z=860.36\left(\mathrm{M}^{+}\right)$

f) 2,2',7,7'-Tetrakis-(biphenyl-3-yl)-9,9' 'spirobifluorene, $m, p-\operatorname{Sp} 6 \Phi(6)$ :

Coupling reagent: pinacol ester of (3-phenyl)phenyl boronic acid, eluent $\mathrm{CH}_{2} \mathrm{Cl}_{2}$ / hexane 2:1

Yield: $51 \%$ (purity $>99 \%$ )

${ }^{1} \mathrm{H}-\mathrm{NMR}: \delta=7.47(\mathrm{~d}, J=7.8 \mathrm{~Hz}, 4 \mathrm{H}), 7.36(\mathrm{~m}, 8 \mathrm{H}), 7.33(\mathrm{~m}$, $8 \mathrm{H}), 6,99(\mathrm{~m}, 20 \mathrm{H}), 6.92(\mathrm{~m}, 4 \mathrm{H}), 6.57(\mathrm{~d}, J=1.24 \mathrm{~Hz}, 4 \mathrm{H})$
${ }^{13}$ C-NMR: $\delta=148.8,141.2,140,7,140.5,139.7,130.5,130.3$, $129.6,127.6,127.4,127.2,126.4,125.1,119.2,65.9$

MS-MALDI: $m / z=925.48\left(\mathrm{M}^{+}\right)$

\section{References}

[1] N. Johansson, J. Salbeck, J. Bauer, F. Weissörtel, P. Bröms, A. Andersson, and W. R. Salaneck, "Solid-state amplified spontaneous emission in some spiro-type molecules: a new concept for the design of solid-state lasing materials," Adv. Mater. 10, 1136-1141 (1998).

[2] J. Salbeck, M. Schörner, and T. Fuhrmann, "Optical amplification in spiro-type molecular glasses," Thin Solid Films 417, 20-25 (2002).

[3] T. Spehr, R. Pudzich, T. Fuhrmann, and J. Salbeck, "Highly efficient light emitters based on the spiro concept," Org. Electron. 4, 61-69 (2003).

[4] D. Schneider, T. Rabe, T. Riedl, T. Dobbertin, 0. Werner, M. Kröger, E. Becker, et al., "Deep blue widely tunable organic solid-state laser based on a spirobifluorene derivative," Appl. Phys. Lett. 84, 4693-4695 (2004).

[5] D. Schneider, T. Rabe, T. Riedl, T. Dobbertin, M. Kröger, E. Becker, H.-H. Johannes, et al., "An ultraviolet organic thin-film solidstate laser for biomarker applications," Adv. Mater. 17, 31-34 (2005).

[6] T. Spehr, A. Siebert, T. Fuhrmann-Lieker, J. Salbeck, T. Rabe, T. Riedl, H.-H. Johannes, et al., "Organic solid-state laser based on spiro-terphenyl," Appl. Phys. Lett. 87, 1161103 (2005).

[7] T. Riedl, T. Rabe, H.-H. Johannes, W. Kowalsky, J. Wang, T. Weimann, P. Hinze, et al., "Tunable organic thin-film laser pumped by an inorganic violet laser diode," Appl. Phys. Lett. 88, 241116 (2006).

[8] A. E. Vasdekis, G. Tsiminis, J. C. Ribierre, L. O'Faolain, T. F. Krauss, G. A. Turnbull, and I. D. W. Samuel, "Diode pumped distributed Bragg reflector lasers based on a dye-to-polymer energy transfer blend," Opt. Express 14, 9211-9216 (2006).

[9] Y. Yang, G. A. Turnbull, and I. D. W. Samuel, "Hybrid optoelectronics: a polymer laser pumped by a nitride light-emitting diode," Appl. Phys. Lett. 92, 163306 (2008).

[10] N. Tessler, G. J. Denton, and R. H. Friend, "Lasing from conjugatedpolymer microcavities," Nature 382, 695-697 (1996).

[11] H. Hillmer, "Mikrolaser-Bauelement und Verfahren zu dessen Herstellung," DE10331586B4 (2003).

[12] M. Berggren, A. Dodabalapur, and R. E. Slusher, "Stimulated emission and lasing in dye-doped organic thin films with Forster transfer," Appl. Phys. Lett. 71, 2230-2232 (1997).

[13] D. Schneider, T. Rabe, T. Riedl, T. Dobbertin, M. Kröger, E. Becker, H.-H. Johannes, et al., "Laser threshold reduction in an all-spiro guest-host-system," Appl. Phys. Lett. 85, 1659-1661 (2004).

[14] N. Miyaura, and A. Suzuki, "Palladium-catalyzed cross coupling reactions of organoboron compounds," Chem. Rev. 95, 2457-2483 (1995).

[15] G. E. Jellison, and F. A. Modine, "Parametrization of the optical functions of amorphous materials at the interband region," Appl. Phys. Lett. 69, 371-373 (1996).

[16] M. Abdel-Awwad, H. Luan, F. Messow, T. Kusserow, A. Wiske, A. Siebert, T. Fuhrmann-Lieker, et al, "Optical amplification and photodegradation in films of spiro-quaterphenyl and its derivatives," J. Lumin. 159, 47-54 (2015). 
[17] H. So, H. Watanabe, M. Yahiro, Y. Yang, Y. Oki, and C. Adachi, "Highly photostable distributed feedback-polymer waveguide blue laser using spirobifluorene derivatives," Opt. Mater. 33, 755-758 (2011).

[18] B. Schartel, T. Dammerau, and M. Hennecke, "Photo- and thermooxidative stability of aromatic spiro-linked bichromophoric cross-shaped molecules," Phys. Chem. Chem. Phys. 2, 4690-4696 (2000).

[19] H. Kogelnik, and C. V. Shank, "Stimulated emission in a periodic structure," Appl. Phys. Lett. 18, 152-154 (1971).

[20] K. P. Kretsch, C. Belton, S. Lipson, W. J. Blau, F. Z. Henari, H. Rost, S. Pfeiffer, et al., "Amplified spontaneous emission and optical gain spectra from stilbenoid and phenylene vinylene derivative model compounds," J. App. Phys. 86, 6155-6159 (1999).

[21] H. Nakanotani, S. Akiyama, D. Ohnishi, M. Moriwake, M. Yahiro, T. Yoshihara, S. Tobita, et al., "Extremely low-threshold amplified spontaneous emission of 9,9' spirobifluorene derivatives and electroluminescence from field-effect transistor structure," Adv. Funct. Mater. 17, 2328-2335 (2007).
[22] T. Spehr, Fluoreszenz und Lasertätigkeit in dünnen amorphen Schichten von Spirobifluorenderivaten (PhD thesis, University of Kassel, 2008).

[23] Y.-Z. Huang, Z. Pan, and R.-H. Wu, "Analysis of the optical confinement factor in semiconductor lasers," J. Appl. Phys. 79, 3827-3830 (1996).

[24] T. G. Fox, "Influence of diluent and copolymer composition on the glass temperature of a polymer system," Bull. Am. Phys. Soc. 1, 123-128 (1956).

[25] A. Siebert, Synthese und Charakterisierung neuer symmetrischer und unsymmetrischer Spiro-p-oligophenyle (PhD thesis, University of Kassel, 2010). 